\title{
Enfermagem de Prática Avançada: estratégia para melhorar o cuidado materno-infantil no Brasil
}

Advanced Practice Nursing: a strategy to improve maternal and child care in Brazil Enfermería de Práctica Avanzada: estrategia para mejorar el cuidado materno-infantil en Brasil

Isadora Costa Andriola ${ }^{1}$ ID https://orcid.org/0000-0003-3446-675X

Andréa Sonenberg ${ }^{2}$ id https://orcid.org/0000-0002-6192-2100

Ana Luisa Brandão de Carvalho Lira ${ }^{1}$ io https://orcid.org/0000-0002-7255-960x

\section{Como citar:}

Andriola IC, Sonenberg A, Lira AL. Enfermagem de Prática Avançada: estratégia para melhorar 0 cuidado materno-infantil no Brasil. Acta Paul Enferm. 2020;33:eAPE20190235.

DOI

http://dx.doi.org/10.37689/actaape/2020AR02356

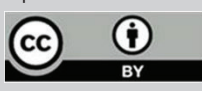

Descritores

Prática Avançada de Enfermagem; Tocologia; Centros de saúde materno-infantil; Acesso aos serviços de saúde; Brasil

Keywords

Advanced practice nursing; Midwifery; Maternalchild health centers; Health Services Accessibility;

Brazi

Descriptores

Enfermería de Práctica Avanzada; Parteria; Centros de salud materno-infantil; Accesibilidad a los servicios de salud; Brasil

\section{Submetido \\ 8 de agosto de 2019 \\ Aceito \\ 16 de março de 2020}

\section{Autor correspondente}

Isadora Costa Andriola

E-mail: dora_andriola@hotmail.com

\section{Resumo}

Objetivo: Investigar como a implementação da Enfermagem de Prática Avançada poderia contribuir para melhorar o status atual dos cuidados maternos e infantis no Brasil.

Métodos: Revisão da literatura realizada em novembro de 2018 a janeiro de 2019 através das seguintes bases de dados: CINAHL, PubMed, MEDLINE, Health Source: Nursing Academic edition e Biblioteca da Universidade PACE. Os dados foram categorizados e analisados considerando o conceito de acesso aos cuidados de saúde. Resultados: De uma revisão de 138 artigos, 10 preencheram os critérios de inclusão e foram revisados para análise. Com base no enfoque utilizado neste estudo, os pesquisadores identificaram duas categorias principais de indicadores sociais para o conceito de acesso: processo e desfecho. Esses temas explorados na análise narrativa são os indicadores de processo e desfecho do sistema de saúde brasileiro, bem como os indicadores de desfecho esperado subsequentes à implementação do papel da enfermeira obstetra.

Conclusão: 0 papel da enfermeira obstetra poderia ajudar o Brasil a melhorar 0 acesso e a qualidade dos cuidados de saúde materno-infantil e o custo-benefício dos cuidados prestados. Esse papel pode contribuir significativamente para um dos principais problemas enfrentados por este país, como desigualdade na distribuição de profissionais de saúde em regiões geográficas.

\section{Abstract}

Objective: This article aims to investigate how the implementation of Advanced Practice Nursing could contribute to improve the current maternal and child care status in Brazil.

Methods: This study is a literature review conducted in November 2018 to January 2019 through the following databases: CINAHL, PubMed, Medline, Health Source: Nursing Academic edition and PACE University Library. The data were categorized and analyzed considering the concept of access to health care.

Results: From a review of 138 papers, 10 met the inclusion criteria and were reviewed for analysis. Based on the framework used in this study, the researchers identified two main categories of social indicators to the access concept: process and outcome. These themes explored in the narrative analysis are the process and outcome indicators of the Brazilian health system, as well as the expected outcome indicators subsequent to implementation of the Certified Nurse-Midwifery role.

Conclusion: The Certified Nurse-Midwife role could help Brazil improve access and quality of maternal and child health care, as well as improve the cost-benefit of care provided. This role may contribute significantly to one of the main problems faced by this country: inequality in the distribution of health professionals in geographical regions. 


\section{Resumen}

Objetivo: Investigar de qué forma la Enfermería de Práctica Avanzada podría contribuir para mejorar el estado actual de los cuidados maternos e infantiles en Brasil.

Métodos: Revisión de la literatura realizada de noviembre de 2018 a enero de 2019 a través de las siguientes bases de datos: CINAHL, PubMed, MEDLINE, Health Source: Nursing Academic edition y Biblioteca de la Universidad PACE. Los datos fueron categorizados y analizados de acuerdo con el concepto de acceso a los cuidados de la salud.

Resultados: De una revisión de 138 artículos, 10 cumplían con los criterios de inclusión y fueron revisados para su análisis. Con base en el enfoque utilizado en este estudio, los investigadores identificaron dos categorías principales de indicadores sociales para el concepto de acceso: proceso y resultado. Estos temas estudiados en el análisis narrativo son los indicadores de proceso y resultado del sistema de salud brasileño, así como los indicadores de resultado esperados subsecuentes a la implementación del papel de la enfermera obstetra.

Conclusión: El papel de la enfermera obstetra podría ayudar a mejorar en Brasil el acceso y la calidad de los cuidados de la salud materno-infantil y el costobeneficio de la asistencia prestada. Este papel puede contribuir significativamente para uno de los principales problemas enfrentados por este país, como la desigualdad en la distribución de profesionales de la salud en regiones geográficas.

\section{Introdução}

Avanços notáveis no setor de saúde ocorreram nas últimas décadas na América Latina e no Caribe, porém as desigualdades sociais e de saúde continuam sendo problemas relevantes. Muitos dos países desta região, incluindo o Brasil, não alcançaram seus objetivos de saúde materna e infantil, resultando em grandes desigualdades. ${ }^{(1)}$ Com uma TMM estimada em 141 por 100.000 nascidos vivos em 1990, segundo o Ministério da Saúde, o Brasil apresentou uma Taxa de Mortalidade Materna (TMM) de 62 por 100.000 nascidos vivos em 2015, que ainda atende aos critérios de alta, e uma Taxa de Mortalidade Infantil (TMI) de 15,1 por 1.000 nascidos vivos no mesmo ano..$^{(2,3)}$

As reduçóes da TMM e da TMI foram dois dos Objetivos de Desenvolvimento do Milênio (ODM) prioritários do Brasil a serem alcançados entre 1990 e 2015.(4) Apesar de haver uma redução relevante da TMM desde 1990, o Governo Federal brasileiro reconheceu que a meta de reduzir a TMM estava entre as mais difíceis de alcançar. ${ }^{(5)} \mathrm{O}$ Brasil alcançou apenas uma redução na TMM de 50\%, em vez de sua meta de reduzi-la em $75 \%$. Isso resultou na classificação do país do quinto país mais lento na busca de reduzir as mortes maternas. ${ }^{(3,6)}$ Em relação à TMI, embora o Brasil tenha atingido uma taxa alvo de 15,7 mortes por 1.000 nascidos vivos, esse indicador é persistentemente alto e ainda há obstáculos a serem superados. ${ }^{(7,8)}$

$\mathrm{Na}$ tentativa de resolver as desigualdades remanescentes na região da América Latina, a ONU pu- blicou sua Agenda 2030 para o Desenvolvimento Sustentável. Neste documento, os Objetivos de Desenvolvimento Sustentável (ODS) para 2030 foram novamente revisados, com o objetivo de diminuir a taxa de prevalência de mortalidade materna e infantil. ${ }^{(3)}$ Um dos desafios para atingir essa meta e resolver essas desigualdades regionais no Brasil é que há distribuições desiguais de médicos em todo o Brasil, deixando áreas remotas relativamente privadas de força de trabalho em saúde adequada. ${ }^{(1)}$

No Brasil, enfermeiros e médicos estão entre os principais prestadores de serviços de saúde que estão diretamente envolvidos na melhoria dos indicadores de saúde de mulheres e crianças. Por outro lado, enfermeiras têm uma distribuição mais uniforme nas diversas regiôes do país em comparação aos médicos. ${ }^{(9)}$ No entanto, o processo de regulamentação dos profissionais de saúde no Brasil é tardio em termos de possibilitar a extensão do escopo da prática a determinadas categorias de prestadores de cuidados de saúde, como a enfermagem. Esse tipo de restrição funciona contra as necessidades da população. Muitos outros países adotaram estratégias para restringir o direito exclusivo da prática a algumas categorias profissionais. ${ }^{(10)}$

A Enfermagem de Prática Avançada (EPA) surge nesse contexto como uma estratégia para melhorar o escopo profissional da prática de enfermagem. A função da enfermeira obstetra, uma das quatro funçôes da EPA, é marcada pelos recursos críticos de foco clínico, defesa do paciente, educação e treinamento, auditoria e pesquisa e consultoria. ${ }^{(11)}$ Com o pré-requisito de um mestrado como entrada no seu 
nível avançado de prática, as enfermeiras obstetras têm como objetivo fornecer assistência e consultoria especializada ao paciente, em sua área de especialidade. ${ }^{(11,12)}$ A OMS recomenda melhorar o escopo da prática dos profissionais de saúde como um meio para melhorar os serviços vitais de assistência à saúde, particularmente em áreas rurais e remotas, e aqueles com escassez absoluta de profissionais de saúde. ${ }^{(13)}$ Para aumentar a força de trabalho e, consequentemente, o acesso à atenção primária à saúde na América Latina e no Caribe, esta organização recomenda a utilização da EPA. ${ }^{(14,15)}$

No contexto brasileiro de saúde materna e infantil, a Rede Cegonha reconhece que uma qualificação profissional está diretamente relacionada à qualidade da assistência e às boas práticas de assistência ao parto e nascimento. Essa política reconhece ainda que a incorporação dessas boas práticas é uma das açóes mais impactantes contra a morbimortalidade materna e infantil. ${ }^{(16)}$ Portanto, a Rede Cegonha recomenda o investimento em treinamento profissional avançado, especialmente para enfermeiros obstétricos, que poderia ser perfeitamente atendido com a implementação do papel da enfermeira obstetra. $\mathrm{O}$ modelo de atendimento da enfermeira obstetra e da Rede Cegonha tem como prioridade um modelo de assistência ao parto que ofereça atendimento humanizado e de qualidade a gestantes, puérperas e recém-nascidos.

Evidências de vários estudos sustentam que a EPA presta mais assistência médica em áreas remotas do que os médicos. ${ }^{(17-19)}$ As evidências também demonstram que a EPA fornece atendimento eficaz e de alta qualidade ao paciente, com a melhoria desejada nos desfechos de saúde, poupança de custos e aumento da cobertura de assistência médica. ${ }^{(19-25)}$ Também é demonstrado que os desfechos dos pacientes dos cuidados prestados pelas enfermeiras obstetras, um dos grupos da EPA, são pelo menos equivalentes e, em alguns casos, melhores do que os desfechos dos cuidados prestados exclusivamente por médicos. ${ }^{(19)}$

Conclui-se que a inclusão do papel da enfermeira obstetra no Brasil poderia promover a obtenção de um melhor acesso aos cuidados e, consequentemente, a melhoria dos desfechos de saúde, ajudando o país a reduzir a TMM e a TMI e, assim, alcançar as metas dos ODS para 2030. A questão de pesquisa é: Como a implementação da EPA, especificamente através das enfermeiras obstetras, pode contribuir para melhorar o atual estado de saúde materno-infantil no Brasil? Este artigo tem como objetivo investigar como a implementação da EPA poderia contribuir para melhorar o status atual dos cuidados de saúde materno-infantil no Brasil.

Para superar os desafios restantes apresentados pelo Brasil após os ODM, os ODS estabeleceram que a TMM global deve ser inferior a 70 por 100.000 nascidos vivos e que as mortes evitáveis de recém-nascidos e crianças com menos de 5 anos devem terminar em 2030. ${ }^{(6)}$ Embora a média brasileira de TMM esteja abaixo dessa meta global, há muitas disparidades entre os estados, alguns deles demonstrando uma TMM acima de 100 mortes por 100.000 nascidos vivos, especialmente nas regióes Norte e Nordeste. ${ }^{(3)}$

A TMM e a TMI estão entre os indicadores mais importantes para demonstrar o estado de saúde de um país. ${ }^{(26)}$ Outros indicadores relevantes do estado de saúde materno-infantil são a taxa de cesárea, baixo peso ao nascer e a taxa de aleitamento materno exclusivo ( $<6$ meses). Fatores de risco adicionais relacionados à TMM e TMI são a cobertura de serviços e a infraestrutura do sistema de saúde, incluindo cobertura pré-natal e capacidade da força de trabalho. ${ }^{(27)} \mathrm{O}$ escopo das estatísticas de cobertura pré-natal revela que, em 2015 , apenas $70,2 \%$ das mulheres brasileiras tiveram acesso a cuidados adequados (ou mais que adequados), pelo menos seis consultas pré-natais. ${ }^{(3)}$ A taxa de cesariana no Brasil, em 2015, registrou 55,5\%. Nesse mesmo ano, os desfechos da saúde de recém-nascidos e bebês também não atingiram as metas estabelecidas: a baixa taxa de peso ao nascer (BPN) no Brasil foi de $8,4 \%$. Em 2017 a prevalência de aleitamento materno exclusivo em crianças até seis meses de idade foi de apenas $38,6 \% .^{(3,28)}$

$\mathrm{Na}$ tentativa de apoiar a conquista dos ODS, em 2011 o governo brasileiro instituiu um programa chamado Rede Cegonha. Este programa é composto por quatro elementos: pré-natal; parto e nascimento; cuidados pós-parto; e atenção integral à saúde da criança. ${ }^{(5)}$ Tem como objetivo prestar atendimento 
qualificado e humanizado, estabelecendo um modelo de prestação de cuidados a gestantes, mães e crianças de até 2 anos de idade. ${ }^{(29)} \mathrm{O}$ pré-natal e o atendimento às mulheres durante o parto são reconhecidos como estratégias relevantes para reduzir o risco de morbidade e mortalidade entre gestantes e crianças e são prioridades para o Sistema Único de Saúde. ${ }^{(3)}$

Para prestar esse atendimento qualificado, é imprescindível que o país lide com seus processos de escassez da força de trabalho em saúde. Para funcionar adequadamente, os sistemas de saúde dependem diretamente de sua força de trabalho. ${ }^{(30)}$ Assim, para obter melhores desfechos e melhorar a cobertura dos serviços de saúde, a força de trabalho em saúde deve ser adequada em seu tamanho e habilidades. ${ }^{(31)}$ A OMS identificou o limiar do índice ODS de 4,45 médicos, enfermeiros e parteiras por 1.000 habitantes como uma densidade mínima indicativa de profissionais de saúde necessários para atingir as metas globais até 2030. ${ }^{(31)}$ Apesar de uma inadequação dessa proporção, o governo brasileiro estima que os médicos aumentem da atual 1,7 por 1.000 habitantes para uma proporção entre 2,3 e 3,5 por 1.000 habitantes nos próximos doze anos. Enfermeiras devem aumentar de 0,7 para uma proporção de 2,4 para 4,0 por 1.000 habitantes. ${ }^{(10)}$

Como ODS prioritário para 2030, o Brasil também deve diminuir sua disparidade na distribuição de profissionais de saúde entre a proibição de urinar e as áreas rurais. ${ }^{(32)}$ Países como o Brasil, com desafios de recursos humanos, precisarão desenvolver estratégias apropriadas, sustentáveis e econômicas para eliminar a escassez baseada nas necessidades. ${ }^{(31)}$ Eles devem considerar inovaçóes em seus modelos de cuidados e estratégias educacionais para incluir provedores, como enfermeiros e enfermeiros de prática avançada, a serem integrados ao sistema de Atenção Primária à Saúde. ${ }^{(33,34)}$

Com relação à saúde materna e infantil, um artigo seminal relata que os cuidados com a enfermeira obstetra resultam em: taxas mais baixas de cesariana; maior chance de partos vaginais; menores taxas de uso de analgesia; menos partos instrumentais; taxas comparáveis de baixo peso ao nascer; e maiores taxas de aleitamento materno, quando comparados aos cuidados prestados pelos médicos.
(19) Considerando os desfechos positivos dos cuidados com a enfermeira obstetra experimentados e comprovados em outros países, e dadas as disparidades de saúde acima mencionadas, o Brasil deve considerar fortemente a implementação desse papel da EPA, como uma estratégia para melhorar o acesso a cuidados materno-infantis de alta qualidade e melhorar desfechos de saúde.

\section{Enfoque conceitual}

A EPA na América Latina e no Caribe é um novo conceito. ${ }^{(35)}$ Para orientar o processo de implementação dessa função, será aplicado o processo, desenvolvimento e implementação de funçóes da EPA, centrada no paciente e centrada no paciente, para o desenvolvimento, implementação e avaliação de funçóes, bem conhecidos como o enfoque do PEPPA. ${ }^{(36)}$ Esse enfoque é reconhecido como a melhor prática para a introdução da EPA e mais de 16 países a implementaram com sucesso. ${ }^{(37)} \mathrm{O}$ enfoque do PEPPA articula nove etapas e estratégias para orientar questóes específicas de implementação da função da EPA, criando um processo flexível e iterativo, conforme ilustrado na figura abaixo (Figure 1).

$\mathrm{O}$ primeiro passo do Enfoque PEPPA ajuda a identificar barreiras e orientar o processo de implementação. ${ }^{(35)}$ Esta etapa também identifica as populaçóes de pacientes como foco das atividades nas etapas subsequentes e estabelece o escopo do processo sob diferentes perspectivas. ${ }^{(36)}$ Assim, para facilitar a implementação e superar os obstáculos inerentes a esse processo, o Enfoque PEPPA apresenta estratégias orientadas à saúde, focadas no paciente, participativas e direcionadas às partes interessadas. ${ }^{(36)}$

Os conceitos relacionados às políticas de saúde são intrínsecos às estratégias apresentadas no Enfoque PEPPA. Na tentativa de conectar o processo de implementação de uma função da EPA aos desfechos de saúde desejados, o conceito de acesso à assistência será abordado. ${ }^{(38)} \mathrm{O}$ acesso aos cuidados pode ser conceituado como procedente dos objetivos das políticas de saúde, através das características do sistema de saúde e da população que se esforça para alcançar melhores desfechos em saúde. Esses 


\section{Enfoque PEPPA}

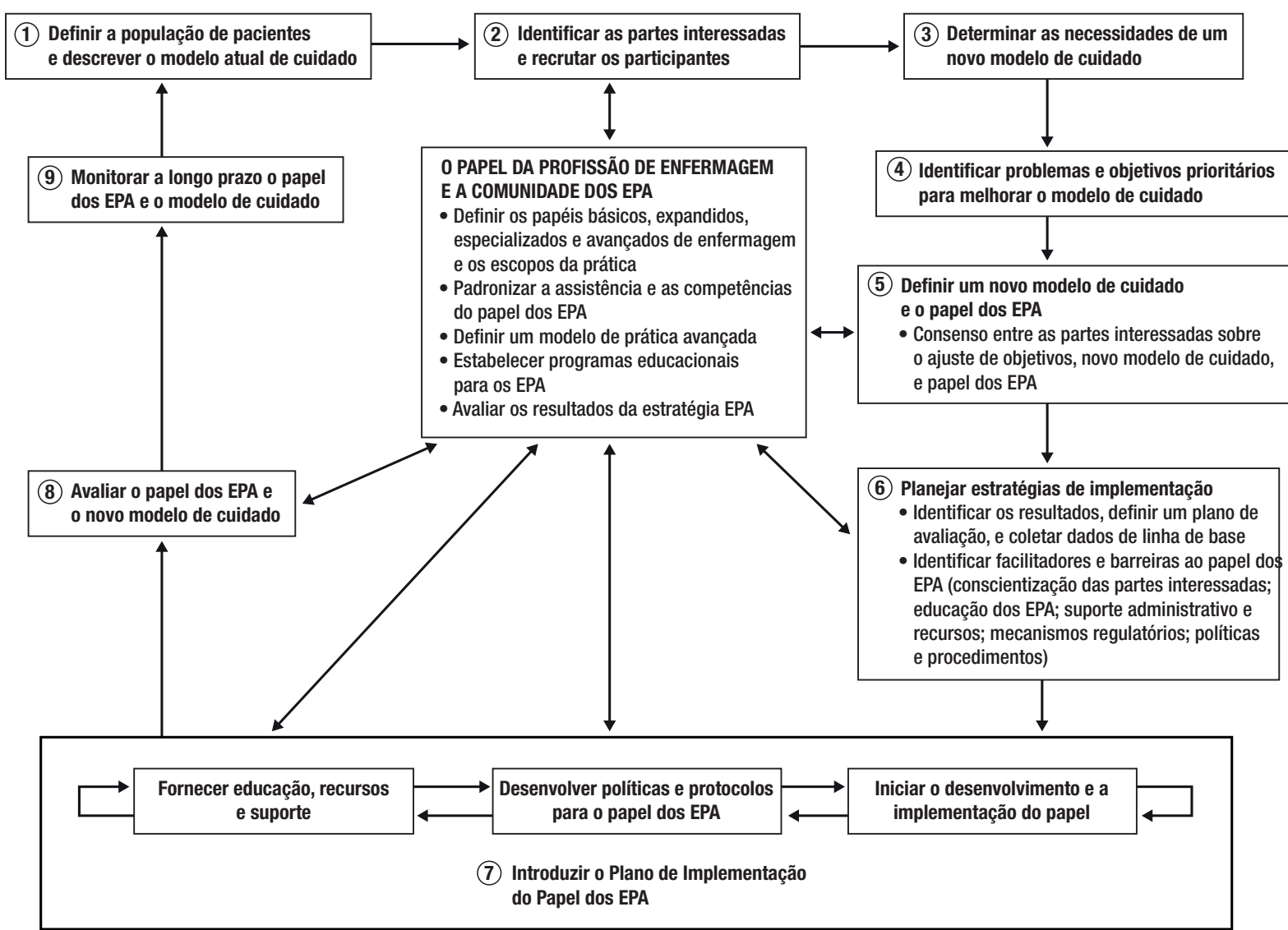

Fonte: Bryant-Lukosius D, \& DiCenso A. Enfoque PEPPA (Participatory, Evidence-based, Patient-focused Process) para o desenvolvimento, implementação e avaliação de funções de Enfermagem de Prática Avançada. J Adv Nurs. 2004; 48(5):530-40.(36)

Figura 1. Enfoque PEPPA

desfechos são a utilização real dos serviços de saúde e a satisfação do consumidor com esses serviços. ${ }^{(38)}$ A Figura 2 demonstra como o enfoque é projetado.

Os autores que enquadraram o conceito de acesso à assistência consideram a política de saúde como fundamento. As políticas de saúde são projetadas para afetar as características do sistema de saúde e a população em risco para obter melhores desfechos. ${ }^{(38)}$ Esses desfechos podem ser entendidos como as mudanças desejadas na utilização dos serviços de saúde e na satisfaçáo dos consumidores. Em outras palavras, o acesso à assistência médica resulta de interaçôes entre indicadores de processo (características da população e características do sistema de saúde) e indicadores de desfecho (satisfaçáo do consumidor e utilização de serviços de saúde), tendo a política de saúde como ponto de partida. No

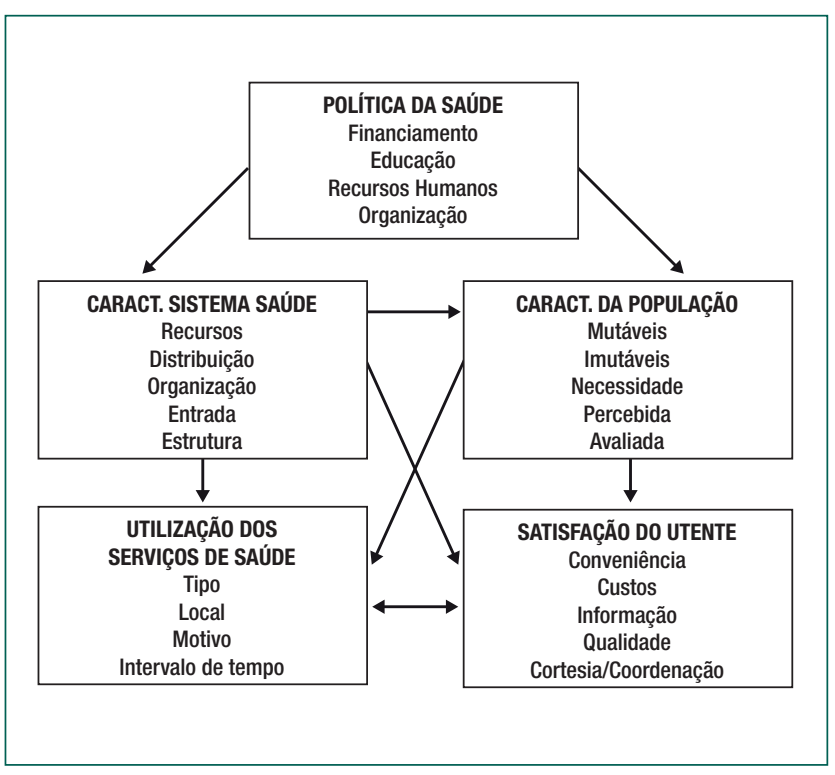

Fonte: Aday LA, Andersen R. Enfoque para o estudo do acesso aos cuidados médicos. Health Serv Res. 1974 Fall; 9(3): 208-220.

Figura 2. Enfoque para o estudo do acesso(38) 
contexto deste estudo, espera-se que a implementação do papel da EPA das enfermeiras obstetras afete positivamente os maus desfechos de saúde materna e infantil no Brasil e a ineficácia do sistema de saúde para alcançar um melhor uso do sistema de saúde e maior satisfação do usuário. ${ }^{(38)}$

Assim, usando essa premissa básica, a política de saúde será o ponto de partida para as mudanças desejadas. ${ }^{(38)}$ De acordo com isso, um dos principais objetivos da implementação da EPA é aumentar o acesso à saúde e melhorar a qualidade da assistência e saúde. ${ }^{(39)}$ Portanto, para informar este estudo, o Enfoque PEPPA é aplicado no contexto do conceito de acesso à assistência ${ }^{(38)}$ para articular a discussão de como a implementação de um papel na EPA poderia potencialmente melhorar a maternidade brasileira. resultados de saúde infantil. $\mathrm{O}$ primeiro passo do Enfoque PEPPA faz parte dessa discussão, ao mesmo tempo em que promove uma compreensão das necessidades da população e como o sistema de saúde e um novo modelo de atendimento podem ser introduzidos.

\section{Métodos}

Este estudo é uma revisão da literatura sobre o status da saúde materno-infantil no Brasil e como o papel da EPA nas enfermeiras obstetras pode contribuir para melhorar os indicadores de saúde nessa populaçáo. Foi construído um protocolo de estudo para organizar o processo de revisão, que incluiu o tema da pesquisa, o objetivo, as questóes da pesquisa, as estratégias de pesquisa como bancos de dados selecionados, os termos do $\mathrm{MesH}$ e as estratégias de combinação, os critérios de inclusão e exclusão e as estratégias para análise dos dados.

Para obter o entendimento pretendido neste estudo, três questóes nortearam o processo de pesquisa na literatura: Como o sistema de saúde brasileiro está organizado no contexto da atenção materna e infantil? Qual é o estado atual de saúde materno-infantil no Brasil? Como a EPA pode ajudar a alcançar melhores desfechos em saúde materna e infantil? A pesquisa foi realizada em novembro de 2018 a janeiro de 2019, através dos seguintes ban- cos de dados: CINAHL, PubMed, MEDLINE, Health Source: Nursing Academic edition e Biblioteca da PACE University. Além disso, foi realizada uma busca secundária através das referências dos estudos selecionados. As bases de dados foram selecionadas de acordo com a possibilidade dos desfechos contemplarem temas específicos da EPA, o que não é uma realidade em todos os países do mundo. A busca eletrônica foi limitada aos estudos realizados nos cinco anos anteriores (2015-2019), para fornecer uma visão atual do estado de saúde no Brasil.

O processo de busca foi orientado pelos seguintes termos da MesH: Acesso aos cuidados de saúde; Indicadores de qualidade em saúde; Serviços de Saúde Materno-Infantil; Saúde Materna; Enfermagem de Prática Avançada e as seguintes palavras-chave: Desfechos do Paciente; Obstetrícia; Brasil. As estratégias de pesquisa combinada usaram AND e OR como operadores booleanos da seguinte maneira. Estratégia de busca combinada 1: (Acesso aos Cuidados de Saúde OR Indicadores de Qualidade dos Cuidados de Saúde) AND (Serviços de Saúde Materna OR Saúde Materna) AND Brasil; e Estratégia de busca combinada 2: Obstetrícia AND Desfechos do Paciente. A seguinte estratégia de busca combinada não obteve nenhum desfecho: Enfermagem de Prática Avançada AND Saúde Materna AND Desfechos do Paciente. A primeira combinação resultou em 34 estudos e o segundo 104 estudos que seriam analisados de acordo com os critérios descritos abaixo.

Os critérios de inclusão são artigos completos publicados nas bases de dados selecionadas, que abordam o tema do estudo e são redigidos em português, inglês ou espanhol. Foram excluídos os artigos que não respondiam às questóes norteadoras deste estudo e não estavam disponíveis eletronicamente, além de publicações editoriais, cartas ao editor, resumos, revisóes e opiniâo de especialistas. Um pesquisador realizou a pesquisa e, em seguida, um segundo pesquisador a revisou.

Os trabalhos foram selecionados após duas leituras. A primeira foi uma leitura exploratória, com a intenção de conhecer o conteúdo do material e fazer uma seleção preliminar. A partir dessa etapa, restaram 18 artigos. A segunda leitura foi interpretativa, 
com o objetivo de reafirmar se o estudo atendia aos critérios de seleção e entender os fatores que poderiam ser alterados pela implementação de um papel da EPA na realidade brasileira. A experiência dos pesquisadores em ambas as realidades, em um país com e sem EPA, também é levada em consideração. A partir desta etapa, 10 artigos foram selecionados para a análise final. A figura abaixo mostra em detalhes os desfechos para as estratégias de pesquisa em cada banco de dados nas três etapas (Figura 3).

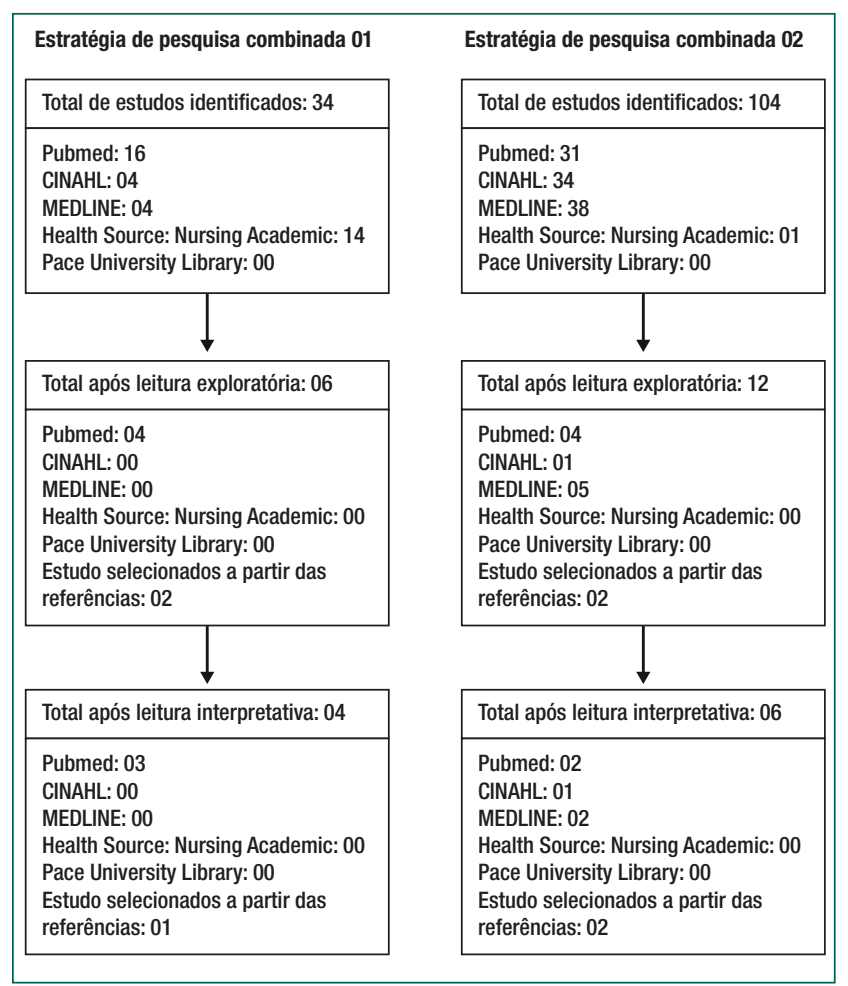

Figura 3. Diagrama do processo de revisão de literatura

Os dados coletados foram organizados em tabelas e as seguintes informaçóes foram extraídas dos estudos: título, autores e ano de publicação; Sistema de saúde brasileiro em cuidados maternos e infantis (rede, estrutura e processos); resultados de saúde no cuidado materno-infantil no contexto brasileiro; resultados de saúde nos cuidados maternos e infantis nos países em que o papel da enfermeira obstetra é implementado. $\mathrm{Na}$ etapa seguinte, os dados foram categorizados e analisados considerando o conceito de acesso à assistência à saúde, que os dividiu em duas categorias principais: processo e indicadores de desfecho, conforme definido na seção que apresenta o arcabouço conceitual. ${ }^{(38)}$
Os indicadores de desfecho também foram divididos em resultados reais e esperados após a implementação do papel da EPA no contexto brasileiro, uma vez que isso não é uma realidade, mas é uma expectativa para este país, com base na experiência de outros países. Os objetivos da política de saúde, considerados o ponto de partida desse conceito, são considerados aqui como o processo de implementação da EPA. ${ }^{(38)}$ Assim, a análise desses dados foi um processo interpretativo, baseado em uma estrutura conceitual.

A discussão desses achados inclui sua posição no Enfoque PEPPA, mais especificamente em sua primeira etapa, em relação às características e necessidades da população para implementar um papel da EPA no Brasil, de acordo com esse arcabouço. Assim, o passo dado neste estudo também é o começo desse processo. É parte de um estudo mais amplo que visa incentivar o processo de implementação da EPA no Brasil, "Proposta de implementação de prática avançada no contexto da enfermagem obstétrica brasileira”, para a qual um dos autores foi aos Estados Unidos da América para analisar seu contexto de prática.

\section{Achados}

A busca resultou em 138 artigos relevantes para a questão de pesquisa. Outros 03 trabalhos foram identificados através de uma busca secundária nas listas de referência dos trabalhos selecionados para a fase interpretativa do estudo. $\mathrm{O}$ número de artigos foi reduzido na etapa de triagem para 18, resultando em um total de 10 sendo analisados na etapa final. A partir desses estudos, os pesquisadores extraíram indicadores que caracterizam o sistema de saúde brasileiro e a rede de atenção materna e infantil; os indicadores que caracterizam os desfechos de saúde no campo da maternidade brasileira; e os indicadores mostrados pelos países onde o cuidado materno e infantil já é fornecido pelas enfermeiras obstetras. Quanto à caracterização dos estudos encontrados, para a estratégia de busca combinada 01 , que busca entender a situação da saúde materna e infantil no Brasil e no sistema de saúde brasileiro, 100\% 
Quadro 1. Processo e indicadores de desfecho relacionados ao Sistema Único de Saúde e indicadores de desfecho esperados após a implementação da função das enfermeiras obstetras

\begin{tabular}{|c|c|c|}
\hline \multirow{2}{*}{$\begin{array}{l}\text { Indicadores de processo } \\
\text { Características do sistema de saúde e população em risco }\end{array}$} & \multicolumn{2}{|c|}{ Indicadores de desfecho } \\
\hline & $\begin{array}{l}\text { Utilização de serviços de saúde e satisfação do } \\
\text { consumidor }\end{array}$ & $\begin{array}{l}\text { Desfechos esperados após a implementação da função } \\
\text { das enfermeiras obstetras }\end{array}$ \\
\hline $\begin{array}{l}\text { Acesso gratuito à saúde fornecido pelo Sistema Único de } \\
\text { Saúde. }{ }^{(40)}\end{array}$ & \multirow[t]{2}{*}{ Altas taxas de intervenção no trabalho de parto e parto. ${ }^{(41,42)}$} & \multirow{2}{*}{$\begin{array}{l}\text { Desfechos clínicos melhores ou equivalentes e menores taxas } \\
\text { de intervenções para os cuidados prestados pelas enfermeiras } \\
\text { obstetras em comparação aos cuidados prestados pelos } \\
\text { médicos. } \text {. }^{(19,43,45)}\end{array}$} \\
\hline 0 sistema de saúde cobre a maioria da população brasileira. ${ }^{(40)}$ & & \\
\hline $\begin{array}{l}0 \text { sistema de saúde enfrenta problemas de subfinanciamento. } \\
\text { (40) }\end{array}$ & \multirow[t]{2}{*}{ Altas taxas de cesariana. ${ }^{(41,42)}$} & \multirow[t]{2}{*}{$\begin{array}{l}\text { Comprova-se que a satisfação do paciente é maior com os } \\
\text { cuidados prestados pelas enfermeiras obstetras. }{ }^{(43)}\end{array}$} \\
\hline $\begin{array}{l}0 \text { Programa Saúde da Família expandiu os cuidados primários } \\
\text { de saúde para as áreas mais pobres, mas as desigualdades } \\
\text { permanecem. } \text {. }^{(41,42)}\end{array}$ & & \\
\hline $\begin{array}{l}\text { Dificuldades no acesso aos cuidados de saúde em áreas } \\
\text { remotas e rurais. }{ }^{(41,42)}\end{array}$ & \multirow[t]{2}{*}{ Nivel de boas práticas obstétricas abaixo da expectativa. ${ }^{(28,42)}$} & \multirow[t]{2}{*}{$\begin{array}{l}\text { A abordagem aos cuidados prestados pelas enfermeiras } \\
\text { obstetras é centrada no paciente. }{ }^{(44)}\end{array}$} \\
\hline $\begin{array}{l}\text { A Rede Cegonha garante atendimento integral no campo de } \\
\text { cuidados maternos e infantis. }{ }^{(41)}\end{array}$ & & \\
\hline A cobertura pré-natal e o parto são quase universais. ${ }^{(8,41)}$ & \multirow[t]{2}{*}{ Deficiências na qualidade da assistência à gestante. ${ }^{(8)}$} & \multirow{2}{*}{$\begin{array}{l}\text { Os desfechos neonatais dos cuidados prestados pelas } \\
\text { enfermeiras obstetras são comparáveis aos cuidados } \\
\text { prestados pelos médicos. }{ }^{(19,45)}\end{array}$} \\
\hline Falta de organização na rede de atenção à saúde. ${ }^{(8)}$ & & \\
\hline Falta de suprimentos e infraestrutura do sistema de saúde. ${ }^{(29,42)}$ & \multirow[t]{2}{*}{ Elevada taxa de mortalidade materna..$^{(8,41)}$} & \multirow[t]{2}{*}{ Maiores taxas de aleitamento materno. ${ }^{(19)}$} \\
\hline Má distribuição de profissionais de saúde em todo o país. ${ }^{(40,41)}$ & & \\
\hline Desigualdades socioeconômicas. ${ }^{(42)}$ & Taxa de mortalidade infantil acima do nível ideal. ${ }^{(8,41)}$ & $\begin{array}{l}\text { Custos de saúde menores ou equivalentes quando } \\
\text { comparados à assistência prestada pelos médicos. }{ }^{(21,45)}\end{array}$ \\
\hline
\end{tabular}

dos manuscritos são estudos desenvolvidos naquele país. Com relação à estratégia de busca combinada 02 , que trata dos cuidados prestados pelas parteiras, 03 desses estudos foram desenvolvidos nos Estados Unidos da América (50\%) e 01 em cada um dos seguintes países: Austrália, Cingapura e Irlanda (16,6\% cada).

Com base no conceito de acesso à assistência $^{(38)}$, os indicadores encontrados nesses estudos foram agrupados em processo e indicadores de desfecho. Esses indicadores podem medir aspectos relevantes do acesso aos cuidados. A política de saúde é projetada para afetar as características do sistema de saúde e da população em risco (indicadores de processo), a fim de alterar a utilização dos serviços de saúde e a satisfação dos consumidores com esses serviços (indicadores de desfecho). Fora isso, a política de saúde pode ser vista em três das etapas do Enfoque PEPPA: 1. descrevendo um modelo atual de assistência; 2 . determinar a necessidade de um novo modelo de atendimento; e 3. identificar problemas prioritários, abordados neste estudo. ${ }^{(39)}$

O Quadro 1 apresenta os indicadores de processos e desfechos encontrados nos 10 artigos investigados nesta pesquisa bibliográfica.

\section{Discussão}

A política de saúde surge como o ponto de partida quando se pretende fazer alteraçóes ou melhorias no acesso à saúde de uma população. ${ }^{(38)}$ Assim, é necessário estabelecer políticas regulatórias da força de trabalho para facilitar a introdução do papel do enfermeiro de prática avançada, particularmente o da enfermeira obstetra, para o sistema de saúde brasileiro. Entre a América Latina e o Caribe, o Brasil foi reconhecido como um dos lugares mais suscetíveis de implementar a EPA. Entre os principais motivos, havia um número crescente de programas de graduação e pós-graduação e a base legal focada na autonomia dos profissionais de enfermagem e na relevância da prática de enfermagem. ${ }^{(46)}$ Para apresentar essas facilidades para implementar a EPA neste país, o Enfoque PEPPA oferece um guia estratégico para estruturar e apoiar esse processo. ${ }^{(25,36)} \mathrm{O}$ primeiro passo dessa estratégia, "definindo a população de pacientes e descrevendo o atual modelo de atendimento", é delineado ao longo deste estudo e será apoiar a realização das seguintes etapas, por meio de tentativas de reforma no contexto sociopolítico do Brasil. ${ }^{(36)}$

Ser enfermeiro obstetra significa ser um prestador de cuidados primários de saúde para mulheres e re- 
cém-nascidos. Prestar atenção primária nesse contexto é fornecer serviços de saúde integrados e acessíveis; atender à maioria das necessidades de saúde dessa população; e praticar no contexto da família e da comunidade, em parceria com a gestante. Ao praticar em um nível avançado, enfermeiras obstetras assumem a responsabilidade pelo fornecimento e encaminhamento a serviços de saúde adequados, incluindo a prescrição, administração e dispensação de agentes farmacológicos. ${ }^{(47)}$ As enfermeiras obstetras brasileiros trabalham com uma filosofia muito semelhante, especialmente considerando que este país possui os cuidados primários de saúde como base de seu sistema de saúde. Apesar de não terem uma prática avançada reconhecida, essas enfermeiras obstétricas podem trabalhar no parto e parto, de uma perspectiva integrada e humanizada, proporcionando cuidados seguros, baseados em evidências científicas. ${ }^{(48)}$

Os indicadores de processo identificados neste estudo, relacionados à morbimortalidade materna e infantil, apresentam um contexto que demonstra que o Brasil ainda precisa abordar, entre outros fatores, os seguintes: desigualdades relacionadas ao desenvolvimento socioeconômico; distribuição desigual de profissionais de saúde nas regióes do país; qualidade do pré-natal; intervençóes excessivas no trabalho de parto e parto; limitaçôes e ineficiências nos níveis organizacional e da rede de saúde; má gestão de recursos financeiros e restrições orçamentárias. ${ }^{(8,41,42)}$ Assim, de acordo com o contexto mencionado acima, reunindo o problema evidenciado no contexto da saúde materno-infantil no Brasil com a proposta das enfermeiras obstetras, é possível inferir que esses indicadores poderiam ser direta e positivamente afetados com a implementação dessa avançada função.

A EPA se destaca por sua capacidade de preencher lacunas de profissionais de saúde em áreas rurais e remotas. ${ }^{(13,25)}$ Isso pode ser tomado como uma das principais razóes para um país implementar um papel da EPA de alcançar as necessidades da população promovendo a saúde, prevenindo a morbidade e reduzindo as taxas de mortalidade nas áreas em que são privados desse direito básico. Para atender a essas necessidades e na tentativa de obter melhores desfechos, muitas enfermeiras com diploma de bacharel no Brasil e em outros países da América Latina trabalham além do seu esco- po de prática, mas sem o benefício da educação formal e da regulamentação profissional. ${ }^{(35)}$ Enfermeiras tendem a estar presentes nessas áreas remotas mais do que os médicos, que geralmente procuram grandes áreas urbanas onde podem realizar subespecialidades e trabalhar em grandes centros de saúde. Assim, enfermeiros podem efetivamente contribuir para o desenvolvimento de sistemas de saúde, especialmente se seu potencial for reconhecido, como quando o escopo da prática é ampliado. ${ }^{(14)}$

Enfermeiras obstetras têm uma prática baseada em uma perspectiva holística e humanizada, atendendo às necessidades das mulheres como seres humanos biopsicossociais inseridos em um contexto complexo. Além disso, eles têm uma visão natural e fisiológica do processo de nascimento e parto. ${ }^{(47)}$ Essa maneira de entender a gravidez, o nascimento e o parto pode ser responsável pela solução do problema de altos índices de intervençôes desnecessárias, como acontece no Brasil. ${ }^{(8,28,41,42)}$ Além disso, é extremamente relevante considerar o fato de que esses profissionais possuem conhecimento técnico e científico suficiente para promover uma prática clínica precisa e resolutiva, que objetiva fornecer uma resposta imediata à mulher que procura atendimento. Isso pode incluir a capacidade de realizar uma avaliação clínica avançada, prescrever medicamentos e solicitar exames, por exemplo.

O Reino Unido (Reino Unido) possui o sistema de saúde pública mais antigo do mundo, o National Health Service (NHS), e pode ser considerado um exemplo para o Brasil. Nesse país, a gravidez e a assistência à mulher no pré-natal, trabalho de parto, parto e pós-parto são abordadas como um processo fisiológico e não como uma doença. Portanto, o parto natural é uma competência em obstetrícia e os médicos são chamados, se necessário, em condições mais específicas e complexas que se apresentam ao longo deste processo. Esse modelo de atendimento pode tornar o sistema mais eficaz e também é responsável por aumentar a qualidade do atendimento prestado. A Holanda, a Nova Zelândia e os países escandinavos também obtêm benefícios com o modelo de assistência em obstetrícia, conhecido por diminuir as taxas de cesarianas e menos intervençôes desnecessárias durante o trabalho de parto e parto. ${ }^{(49)}$ 
Ao implementar o modelo liderado por enfermeiras obstetras para a prestação de cuidados obstétricos, o Brasil poderia beneficiar o sistema de saúde atual, melhorando os desfechos dos pacientes ao mesmo tempo em que diminui os custos. ${ }^{(19,21,45)}$ Assim, o papel da EPA poderia fornecer os cuidados necessários como uma modalidade de alta qualidade, segura e de custo relativamente baixo do profissional de saúde para a população obstétrica. Portanto, o uso da EPA, especificamente as enfermeiras obstetras neste contexto, poderia melhorar o acesso à assistência médica no Brasil.

\section{Conclusão}

A implementação dos papéis da EPA no contexto brasileiro, especificamente a das enfermeiras obstetras, poderia ajudar o país a melhorar seus indicadores de desfechos relacionados ao acesso à saúde na população materna-infantil. Esse processo precisa ser guiado pelo Enfoque Estratégico do PEPPA, incluindo mudanças na política de saúde, e é urgente e necessário, dadas as características do sistema de saúde e da população em risco. Assim, com base nas evidências geradas pelas experiências de outros países, o Brasil poderia melhorar o acesso à saúde materno-infantil, melhorando o acesso à qualidade da assistência e melhorando o custo-benefício da assistência prestada. Além disso, o investimento na força de trabalho qualificada de enfermagem existente pode contribuir significativamente para um dos principais problemas enfrentados por este país: desigualdade na distribuição de profissionais de saúde em regióes geográficas. $\mathrm{O}$ desafio de aplicar os achados deste estudo é identificar a implementação mais eficiente das evidências geradas por outros países, que possuem diferentes estruturas do sistema de saúde, e traduzir esse conhecimento para a realidade brasileira. Para estudos futuros, a América Latina e o Caribe devem se esforçar para implementar as etapas de enfoque do PEPPA, para avançar na direção dessa implementação de função desejada. Engajar enfermeiras e partes interessadas nessa missão é o atual desafio brasileiro.

\section{Agradecimentos}

À CAPES, pelo financiamento do projeto, através de uma doação do Programa de Doutorado Sanduíche no Exterior (PDSE) à autora Isadora Costa Andriola.

\section{Referências}

1. Pan American Health Organization (PAHO). Health in the Americas. Washington (D.C.): Pan American Health Organization; 2017.

2. World Health Organization (WHO). Maternal Health and Safe Motherhood Program. Perinatal mortality: a listing of available information. Geneva: World Health Organization; 1996.

3. Brasil. Ministério da Saúde. Secretaria de Vigilância em Saúde. Departamento de Vigilância de Doenças e Agravos não Transmissíveis e Promoção da Saúde. Saúde Brasil 2017: uma análise da situação de saúde e os desafios para o alcance dos objetivos de desenvolvimento sustentável. Brasília (DF): Ministério da Saúde; 2018.

4. United Nations (UN). The United Nations Millennium Declaration. New York: United Nations; 2000.

5. Brasil. Secretaria de Governo. Guia de apoio para o alcance das metas - Agenda de Compromissos dos Objetivos de Desenvolvimento do Milênio - Governo Federal e Municípios 2013-2016. Brasília (DF): Secretaria de Governo; 2013.

6. United Nations. Transforming Our World: The 2030 Agenda for Development Sustainable. New York: United Nations; 2015.

7. França E, Teixeira R, Ishitani L, Duncan BB, Cortez-Escalante JJ, Morais Neto OL, et al. Causas mal definidas de óbito no Brasil: método de redistribuição baseado na investigação do óbito. Rev Saude Publica. 2014;48(4):671-81.

8. Leal MD, Bittencourt SD, Torres RM, Niquini RP, Souza PR Jr. Determinants of infant mortality in the Jequitinhonha Valley and in the North and Northeast regions of Brazil. Rev Saude Publica. 2017;51(0):12.

9. Brasil. Ministério da Saúde. Secretaria de Gestão do Trabalho e Educação em Saúde (SGTES). Departamento de Gestão e da Regulação do Trabalho (DGERTS): CONPROF - Conselhos Profissionais e Base Demográfica do IBGE; 2010.

10. Dal Poz MR, Perantoni CR, Girardi S. Formação, mercado de trabalho e regulação da força de trabalho em saúde no Brasil. In: Fundação Oswaldo Cruz. A saúde no Brasil em 2030 - prospecção estratégica do sistema de saúde brasileiro: organização e gestão do sistema de saúde [Internet]. Rio de Janeiro: Fio-Cruz; 2013. Vol. 3. p.187-233. [citado 2020 Mar 15]. Disponível em: http://capacidadeshumanas. org/trajetoriainstitucionaldosus/publicacoes/formacao-mercado-detrabalho-e-regulacao-da-forca-de-trabalho-em-saude-no-brasil/

11. National Council for the Professional Development of Nursing and Midwifery (NCNM). Framework for the Establishment of Clinical Nurse/ Midwife Specialist Posts. Dublin: NCNM; 2004.

12. International Council of Nurses (ICN). The Scope of practice, standards and competencies of the advanced practice nurse. Geneva: ICN; 2008.

13. World Health Organization (WHO). Increasing access to health workers in remote and rural areas through improved retention: global policy recommendations. Geneva: WHO; 2010. 
14. Pan American Health Organization (PAHO). Human Resources for Health: Increasing Access to Qualified Health Workers in Primary Health Care-Based Health Systems. Washington (D.C.): PAHO; 2013.

15. Pan American Health Organization (PAHO). Estratégia para cobertura universal de saúde. In: 154a Sessão do Comitê Executivo. Washington (D.C.): PAHO; 2014

16. Brazil. Ministério da Saúde. Secretaria de Atenção à Saúde. Manual prático para implementação da Rede Cegonha. Brasília (DF): Ministério da Saúde; 2011.

17. Buerhaus PI, DesRoches CM, Dittus R, Donelan K. Practice characteristics of primary care nurse practitioners and physicians. Nurs Outlook. 2015;63(2):144-53.

18. Chin WY, Lam CL, Lo SV. Quality of care of nurse-led and allied health personnel-led primary care clinics. Hong Kong Med J. 2011;17(3):217-30.

19. Newhouse RP, Stanik-Hutt J, White KM, Johantgen M, Bass EB, Zangaro G, et al. Advanced practice nurse outcomes 1990-2008: a systematic review. Nurs Econ. 2011;29(5):230-50.

20. Bryant-Lukosius D, Martin-Misener R. Advanced Practice Nursing: an essential component of country level human resources for health [Internet]. Genebra: ICN; 2016. [ICN Policy Brief 6]. [cited 2020 Mar 15]. Available from: https://www.who.int/workforcealliance/ knowledge/resources/ICN_PolicyBrief6AdvancedPracticeNursing.pdf

21. Casey M, O'Connor L, Cashin A, Smith R, O'Brien D, Nicholson E, et al. An overview of the outcomes and impact of specialist and advanced nursing and midwifery practice, on quality of care, cost and access to services: A narrative review. Nurse Educ Today. 2017; 56:35-40.

22. Kilpatrick K, Kaasalainen S, Donald F, Reid K, Carter N, Bryant-Lukosius D, et al. The effectiveness and cost-effectiveness of clinical nurse specialists in outpatient roles: a systematic review. J Eval Clin Pract. 2014;20(6):1106-23.

23. Kurtzman ET, Barnow BS. A comparison of nurse practitioners, physician assistants, and primary care physicians' patterns of practice and quality of care in health centers [Internet]. Med Care. 2017;55(6):615-22.

24. Ndosi M, Lewis M, Hale C, Quinn H, Ryan S, Emery P, et al. The outcome and cost-effectiveness of nurse-led care in people with rheumatoid arthritis: a multicentre randomised controlled trial. Ann Rheum Dis. 2014;73(11):1975-82.

25. Oldenburger D, Cassiani SHB, Bryant-Lukosius D, Valaitis RK, Baumann A, Pulcini J, Martin-Misener R. Implementation strategy for advanced practice nursing in primary health care in Latin America and the Caribbean. Rev Panam Salud Publica. 2017:41:e40.

26. World Health Organization (WHO). Global Reference List of 100 Core Health Indicators (plus health-related SDGs). Geneva: WHO; 2018.

27. Pan American Health Organization (PAHO). Health Situation in the Americas: Core Indicators 2017. Washington (D.C.): PAHO; 2017.

28. Shekar M, Kakietek J, Dayton Eberwein J, Walters D. Reaching the global target for breastfeeding. In Shekar M, Kakietek OJ, Walters D, Dayton Eberwein J, editors. An investment framework for nutrition: Reaching the global targets for stunting, anemia, breastfeeding, and wasting. Directions in Development-Human Development. Washington (D.C.): World Bank Group; 2017.

29. Almeida KJ, Roure FN, Bittencourt RJ, Santos RM, Bittencourt FV, Gottems LB, et al. Active health Ombudsman service: evaluation of the quality of delivery and birth care. Rev Saude Publica. 2018;52:76.

30. Campbell J, Dussault G, Buchan J, Pozo-Martin F, Guerra Arias M, Leone C, et al. A universal truth: no health without a workforce. Forum report, Third Global Forum on Human Resources for Health, Recife, Brazil. Geneva: Global Health Workforce Alliance and World Health Organization; 2013.
31. World Health Organization (WHO). Health workforce requirements for universal health coverage and the Sustainable Development Goals. Geneva: WHO; 2016.

32. World Health Organization (WHO). Global Strategy on Human Resources for Health: Workforce 2030. Geneva: WHO; 2016.

33. American Association of Nurse Practitioners (AAPN). Nurse practitioner cost-effectiveness. Austin (Texas): AAPN; 2013.

34. O'Hare S. Mid-level providers in a changing healthcare workforce [Internet]. Becker's Hospital Review. 2010; August 17. [cited 2020 Mar15]. Available from: http://www.beckershospitalreview.com/ compensation-issues/mid-level-providers-in-a-changing-healthcareworkforce.html

35. Aguirre-Boza F, Cerón MC, Pulcini J, Bryant-Lukosius D. Implementation strategy for advanced practice nursing in primary health care in Chile. Acta Paul Enferm. 2019;32(2):120-8.

36. Bryant-Lukosius D, Dicenso A. A framework for the introduction and evaluation of advanced practice nursing roles. J Adv Nurs. 2004;48(5):530-40

37. Boyko JA, Carter N, Bryant-Lukosius D. Assessing the spread and uptake of a framework for introducing and evaluating advanced practice nursing roles. Worldviews Evid Based Nurs. 2016;13(4):277-84.

38. Aday LA, Andersen R. A framework for the study of access to medical care. Health Serv Res. 1974;9(3):208-20.

39. Bryant-Lukosius D, Valaitis R, Martin-Misener R, Donald F, Peña LM, Brousseal L. Enfermagem com prática avançada: uma estratégia para atingir cobertura universal de saúde e acesso universal à saúde. Rev Lat Am Enfermagem. 2017;25:e2826

40. Paim J, Travassos C, Almeida C, Bahia L, Macinko J. The Brazilian health system: history, advances, and challenges. Lancet. 2011;377(9779):1778-97.

41. Cassiano MC, Carlucci EM, Gomes CF, Bennemann RM. Saúde materno infantil no Brasil: evolução e programas desenvolvidos pelo Ministério da Saúde. Rev Serv Público (Brasília). 2014;65(2):227-44.

42. França GV, Restrepo-Méndez MC, Maia MF, Victora CG, Barros AJ. Coverage and equity in reproductive and maternal health interventions in Brazil: impressive progress following the implementation of the Unified Health System. Int J Equity Health. 2016;15(1):149.

43. Schober MM. Factors influencing the development of advanced practice nursing in Singapore [Doctoral]. United Kingdom: Sheffield Hallam University; 2013. 243

44. Atsalos C, Biggs K, Boensch S, Gavegan FL, Heath S, Payk M, et al. How clinical nurse and midwifery consultants optimise patient care in a tertiary referral hospital. J Clin Nurs. 2014;23(19-20):2874-85.

45. Altman MR, Murphy SM, Fitzgerald CE, Andersen HF, Daratha KB. The cost of nurse-midwifery care: use of interventions, resources, and associated costs in the hospital setting. womens health issues. 2017;27(4):434-40.

46. Bezerril MS, Chiavone FB, Mariz CM, Sonenberg A, Enders BC, Santos VE. Advanced practice nursing in Latin America and the Caribbean: context analysis. Acta Paul Enferm. 2018;31(6):636-43.

47. American College of Nurse-Midwives (ACNM). Core Competencies for Basic Midwifery Practice. Massachusetts: ACNM; 2012.

48. Amaral RC, Alves VH, Pereira AV, Rodrigues DP, Silva LA, Marchiori GR. The insertion of the nurse midwife in delivery and birth: obstacles in a teaching hospital in the Rio de Janeiro state. Esc Anna Nery. 2019;23(1):e20180218.

49. Norman AH, Tesser CD. Midwives and obstetric nurses in the Brazilian Unified Health System and Primary Health Care: for a systemic and progressive incorporation. Rev Bras Med Fam Comun. 2015;10(34):1-7. 